\title{
Cyclooxygenase-2 inhibition: effects on tumour growth, cell cycling and lymphangiogenesis in a xenograft model of breast
}

\section{cancer}

\author{
NLP Barnes', F Warnberg', G Farnie', D White', W Jiang', E Anderson' and NJ Bundred ${ }^{*, I}$ \\ 'Department of Academic Surgery, South Manchester University Hospital, Research and Education Building 2nd Floor, Southmoor Road, Wythenshawe, \\ Manchester M23 9LT, UK; ${ }^{2}$ The Department of Angiogenesis and Metastasis Research, University of Wales College of Medicine, Cardiff, Wales, UK
}

Cyclooxygenase-2 (COX-2) is associated with poor-prognosis breast cancer. We used a nude mouse xenograft model to determine the effects of COX-2 inhibition in breast cancer. Oestrogen receptor (ER)-positive MCF7/HER2-I 8 and ER-negative MDAMB23 I breast cancer cell lines were injected into nude mice and allowed to form tumours. Mice then received either chow containing Celecoxib (a COX-2 inhibitor) or control and tumour growth measured. Tumour proliferation, apoptosis, COX-2, lymphangiogenesis and angiogenesis were assessed by immunohistochemistry $(\mathrm{HC})$, Western blotting or Q-PCR. Celecoxib inhibited median tumour growth in MCF7/HER2- I $8(58.7 \%, P=0.029)$ and MDAMB23I $(46.3 \%, P=0.0002)$ cell lines compared to control. Cyclooxygenase-2 expression decreased following Celecoxib treatment (MCF7/HER2- I 8 median control 65.3\% vs treated $22.5 \%, P=0.000 \mathrm{I}$ ). Celecoxib increased apoptosis in MCF7/HER2- I 8 tumours (TUNEL $0.52 \%$ control vs $0.73 \%$ treated, $P=0.0004$ ) via inactivation of AKT (median PAKT ${ }^{\text {ser473 }} 57.3 \%$ control vs $35.5 \%$ treated, $P=0.000$ I - confirmed at Western blotting). Q-PCR demonstrated decreased podoplanin RNA (lymphangiogenesis marker) in the MCF7/HER2-I8 - median 2.9 copies treated vs 66.6 control $(P=0.05)$ and MDAMB23I-treated groups - median 160.7 copies vs 0.05 control copies $(P=0.015)$, confirmed at IHC. Cyclooxygenase-2 is associated with high levels of activated AKT ${ }^{\text {ser473 }}$ and lymphangiogenesis in breast cancer. Cyclooxygenase-2 inhibition decreases tumour growth, and may potentially decrease recurrence, by inactivating AKT and decreasing lymphangiogenesis. British Journal of Cancer (2007) 96, 575-582. doi:I0.1038/sj.bjc.6603593 www.bjcancer.com

Published online 6 February 2007

(c) 2007 Cancer Research UK

Keywords: breast; cyclooxygenase-2; apoptosis; lymphangiogenesis

Cyclooxygenase-2 (COX-2) is overexpressed in approximately $70 \%$ of cases of in situ and $60 \%$ of invasive breast cancer (Boland et al, 2004). The COX genes encode for enzymes that catalyse the ratelimiting step of conversion from arachidonic acid to prostaglandins. Two main isoforms, COX-1 and COX-2, were first demonstrated in the early 1990s (Fu et al, 1990; Xie et al, 1991). Cyclooxygenase- 1 is constitutively expressed in most tissues, and is responsible for physiological housekeeping functions such as gastric cytoprotection, regulation of renal blood flow and platelet aggregation (Smith et al, 2000). Cyclooxygenase-2 is an inducible isoform, and is expressed by cells involved in the inflammatory process (Subbaramaiah et al, 1996; Smith et al, 2000) (i.e. macrophages, monocytes, synoviocytes) and also in a wide variety of solid epithelial tumours (Gasparini et al, 2003). The increased expression of COX-2 in tumourigenesis is secondary to multiple activations and as a response to growth factors and oncogenes, including those in the EGFR/HER2/RAS/MAP kinase pathway.

*Correspondence: Professor NJ Bundred, Department of Academic Surgery, South Manchester University Hospital, Research and Education Building 2nd Floor, Southmoor Road, Wythenshawe, Manchester M23 9LT, UK; E-mail: bundredn@man.ac.uk

Received 4 September 2006; revised 14 December 2006; accepted 19 December 2006; published online 6 February 2007
This latter pathway is of particular interest in breast cancer as HER2 is often upregulated in both invasive and in situ disease and is associated with poor prognosis tumours (Slamon et al, 1987; Sposto and Silverstein, 2002). Conversely, COX-2 is rarely expressed in normal tissue (unless in proximity to an area of neoplasia or inflammation). When COX-2 is expressed in normal tissue, it has been found in a subpopulation of normal breast cells with silenced $\mathrm{p} 16^{\mathrm{ink} 4 \mathrm{a}}$ (which have been postulated as precursors of breast cancer) (Crawford et al, 2004).

Studies of transgenic mice engineered to overexpress COX-2 have shown that these mice form mammary tumours earlier and more often than wild-type mice, their mammary glands contain fewer apoptotic cells and show reduced expression of the proapoptotic proteins BAX and $\mathrm{BCl}-\mathrm{x}(\mathrm{L})$ (Liu et al, 2001). Elevated COX-2 expression leads to increased tumour recurrence and decreased survival in invasive breast cancer (Crawford et al, 2004). The recent publication of the Women's Health Initiative results (Harris et al, 2003) suggest that the regular use of nonsteroidal anti-inflammatory drugs (NSAIDs) has a significant chemopreventative effect against developing breast cancer, with a $28 \%$ reduction (relative risk $0.72,95 \%$ CI $0.56-0.91$ ) with regular NSAID use for 10 years or more.

Previous studies have shown significant decreases in various solid tumour growths following the administration of COX-2 inhibitors (Connolly et al, 2002; Howe et al, 2002), although there 
is still a need to clarify the mechanism of action in breast cancer and identify surrogate markers of effect.

The aims of this study were to use a xenograft model of breast cancer to confirm the effect of COX-2 inhibition on tumour growth in vivo, and to subsequently determine the mechanism of action, by studying expression of markers of cell proliferation (Ki67 and p $21^{\text {cipl }}$ ), apoptosis (TUNEL) and AKT activation.

We also aimed to determine whether Celecoxib perturbed the production of the new lymphatic vessels (lymphangiogenesis). It has recently been demonstrated that expression of the lymphangiogenic factor VEGF-C is significantly elevated in cells overexpressing COX-2 ( $\mathrm{Su}$ et al, 2004). The production of new lymphatic vessels by the tumour is a key step in the promotion of cancer metastasis and COX-2 inhibitors have been shown to have antimetastatic activity in a metastatic mouse model (RocheNagle et al, 2004). In addition, markers of tumour endothelial cell formation have been shown to correlate with prognosis in breast cancer (Davies et al, 2004). In this paper, we used podoplanin, a marker of lymphatic endothelial cells to demonstrate changes in lymphangiogenesis following treatment with Celecoxib in our xenograft model.

\section{MATERIALS AND METHODS}

\section{Tumour cell lines}

One oestrogen receptor (ER)-positive and one ER-negative breast cancer cell line were chosen for study. MCF7/HER2-18, is an ER-positive MCF7 cell line which had been stably transfected with HER2 (EGFR negative /HER2 positive/ER positive), a gift from Professor CC Benz, University of California San Francisco, and MDAMB231 is an ER-negative breast cancer cell line which is EGFR positive /HER2 negative. All cells were routinely maintained in Dulbecco's Modified Eagle Medium with $10 \%$ foetal calf serum, $2 \mathrm{~mm}$ glutamine, $100 \mathrm{U}$ penicillin and $100 \mu \mathrm{g}$ streptomycin per $500 \mathrm{ml}$. The cells were incubated at $37^{\circ} \mathrm{C}$ in a humidified environment and passaged at near confluence.

\section{Nude mouse xenograft model}

The experimental design followed a well-established female nude mouse model from our department (Gandhi et al, 2000; Chan et al, 2002). Following ethical approval, 2-4 million cells were injected into each flank of female $\mathrm{BLAB} / \mathrm{c} \mathrm{nu} / \mathrm{nu}$ mice, aged 6-8 weeks, and allowed to form tumours. The tumours were measured with callipers twice weekly (length $\times$ width) and the mice weighed. The mice had free access to feed, water and bedding at all time, and were housed with a $12 \mathrm{~h}$ light/dark cycle in filter top cages, maximum six mice per cage.

Tumour volumes $\left(\mathrm{mm}^{3}\right)$ were calculated by the formula length $\times$ length $\times($ width $/ 2)$. When $>75 \%$ of the tumours were palpable the mice were randomised to receive either chow containing $0.15 \%$ Celecoxib (Celecoxib donated from Pfizer/chow constituted at Purina Mills, USA) or control chow. The tumours continued to be measured and the mice weighed twice weekly. When tumours in either group reached $1 \mathrm{~cm}^{3}$, the experiment was ended in accordance with UK Home Office guidelines and the tumours were harvested. The tumours were then split, with half fixed in $4 \%$ formalin and embedded in paraffin blocks for immunohistochemistry (IHC), and the remainder snap frozen in liquid nitrogen.

\section{Immunohistochemistry}

Three micron sections of the paraffin blocks were cut onto APEScoated slides. The slides were dewaxed in xylene and rehydrated through graded alcohols to PBS. Staining for COX-2 and Ki67 (Boland et al, 2004) was as described previously. All incubations were carried out at room temperature unless otherwise stated. For $\mathrm{p} 21, \mathrm{pAkt}^{\mathrm{ser} 473}$ and podoplanin, endogenous peroxidase activity was blocked by immersing slides in $0.2 \% \mathrm{H}_{2} \mathrm{O}_{2}$ in methanol for $10 \mathrm{~min}$. At TUNEL staining, sections were treated with proteinase $\mathrm{K}$ for $15 \mathrm{~min}$ before quenching endogenous peroxidase activity. Antigen retrieval was performed by immersing slides in citrate buffer ( $\mathrm{pH}$ 6), in a pressure cooker for p21 (120s), pAkt ${ }^{\text {ser473 }}$ (100 s) and podoplanin (120s).

\section{TUNEL}

Sections were incubated in equilibration buffer (Apoptag Kit, Intergen, Norcross, GA, USA) for $1 \mathrm{~h}$. Then working strength TdT enzyme was applied to the sections, which were incubated for $1 \mathrm{~h}$ at $37^{\circ} \mathrm{C}$. Immersing the sections in working strength stop buffer for 10 min halted the reaction. Following washing in PBS, the sections were incubated with anti-digoxigenin conjugate for $30 \mathrm{~min}$.

\section{p21 ${ }^{\text {cip1 }}$}

Sections were incubated with the Mouse-on-Mouse (MOM) kit blocking solution with avidin for $1 \mathrm{~h}$. Following washing in PBS, the sections were incubated for $5 \mathrm{~min}$ with the working solution of MOM dilutent for $5 \mathrm{~min}$, and then incubated with the primary mouse anti-p21 antibody (DAKO M7202 mouse monoclonal) $1: 25$ made up in MOM dilutent with biotin for $60 \mathrm{~min}$. Following washing in PBS, sections were incubated with working MOM kit biotinylated anti-mouse IgG for $30 \mathrm{~min}$.

\section{Phosphorylated $\mathrm{Akt}^{\text {ser473 }}$}

Nonspecific binding was blocked with $10 \%$ normal goat serum in PBS with avidin for $15 \mathrm{~min}$. The sections were then incubated with the primary phosphor-AKT/PKB ${ }^{\text {ser473 }}$ antibody (rabbit polyclonal, Cell Signalling Technology, Danvers, MA, USA, \#9277) $1: 30$ in $10 \%$ normal goat serum with biotin for $2 \mathrm{~h}$, then with the biotinylated goat anti-rabbit secondary $1: 200$ (Vector BA1000) in $10 \%$ normal goat serum for $1 \mathrm{~h}$.

\section{Podoplanin}

Nonspecific binding was blocked by incubating slides in $10 \%$ normal rabbit serum in PBS with avidin for $30 \mathrm{~min}$, the slides were then incubated with the primary monoclonal antibody to podoplanin (ab11936, AbCam) 1:500 made up in 10\% normal rabbit in PBS with biotin for $2 \mathrm{~h}$. After washing in PBS, the biotinylated rabbit anti-hamster secondary was added (1:500 ab6782 AbCam) for $1 \mathrm{~h}$.

For $\mathrm{p} 21, \mathrm{pAkt}^{\mathrm{ser} 473}$, and podoplanin, following the secondary antibody, sections were incubated with Vectastain Elite $A B C$ reagent (Vector, Peterborough, UK) for $30 \mathrm{~min}$. All stainings were visualised with $\mathrm{DAB}$, counter-stained with haematoxylin, slides then dehydrated, cleared and mounted.

\section{IHC scoring}

All scorings were carried out blind to the treatment group. For $\mathrm{Ki} 67, \mathrm{p} 21^{\mathrm{cip} 1}$ and $\mathrm{pAkt}^{\mathrm{ser} 473}$, the percentage of positive nuclei out of at least 1000 counted, was determined over representative tumour areas. At least 3000 cells were counted when determining the TUNEL score, as there were few apoptotic cells in each specimen. Reproducibility and accuracy of the scoring was ensured by repeat scoring and multiple observer scoring of the initial samples. For $\mathrm{pAkt}^{\mathrm{ser} 473}$ the cytoplasmic staining intensities were scored on a rating $0-3+$. The COX-2 cytoplasmic staining was scored on both the percentage of positive cells and the staining intensity for the MCF7/HER2-18. The grading of intensity for each section was determined following reference to sections taken to be 
representative for each grade of intensity; $0=$ no staining, $1=$ mild staining, $2=$ moderate staining, $3=$ strong, $4=$ very strong. At least 1000 cells were also counted for each section, and the percentage of positively staining cells determined, and assigned a numerical score; $1=$ no staining, $2=<10 \%, 3=11-50 \%, 4 \geqslant 50 \%$. It was considered inappropriate to use the intensity score for the MDAMB231 cells, as COX-2 expression was localised to cytoplasmic vesicles, and there was no discernable difference in intensity. Scoring was therefore calculated from the proportion of cells containing cytoplasmic vesicles. All scorings were carried out using a light microscope, grid graticule and cell counter at $\times 400$ magnification. For podoplanin, vessel density was calculated using a Chalkley point graticule. Scoring was taken as the median number of vessels crossing the points, from six separate fields of high vessel density, at $\times 400$ magnification.

\section{Western blotting}

Snap frozen tumour samples were utilised for Western blotting. Crushing the tissue samples in liquid nitrogen created tumour powder. Lysis buffer was then added to create cell lysates, which were left on ice for $40 \mathrm{~min}$ before centrifugation (at 13000 r.p.m. for $10 \mathrm{~min}$ at $4{ }^{\circ} \mathrm{C}$ ) and protein estimation (using the Biorad protein assay solution and a GeneQuant pro machine).

Following the addition of sample buffer to the lysates, $50 \mu \mathrm{g}$ of protein was resolved onto SDS gel and transferred to Hybond membranes using a TRIS/glycine/SDS electrode tank buffer, run overnight. Membranes were blocked with 5\% skimmed milk-TBSTween for $1 \mathrm{~h}$ and then probed with the primary antibodies; pPKB/ $\operatorname{pAKT}^{\text {ser473 }}$ (1:1000 \#9271 Cell Signaling Technology) and then total PKB/AKT (1:1000 \#9272 Cell Signaling Technology) as a loading control.

After washing in TBS-Tween, the membranes were incubated with a donkey anti-rabbit HRP-linked secondary antibody (1:1000 Amersham Biosciences NA934V, Piscataway, NJ, USA) for $1 \mathrm{~h}$. Following thorough washing in TBS-Tween for $6 \times 10 \mathrm{~min}$, the level of specific protein was visualised by chemiluminescence (Supersignal West Dura extended duration substrate; Pierce Biotechnology, Rockford, IL, USA). Both total PKB/AKT and pPKB/AKT ${ }^{\text {ser473 }}$ bring up single bands of $60 \mathrm{kDa}$. Quantification of protein levels was estimated at subsequent densitometry using GeneTools from Syngene version 3.05.

\section{Q-RT-PCR}

The assessment of lymphangiogenesis (podoplanin) and angiogenesis (CD31) was determined by quantitative PCR utilising snapfrozen tumour samples, which were stored in liquid nitrogen until use. Samples were homogenised to extract RNA using RNA-Zol reagent (Abgene homogenisation kit, Epsom, UK) as per manufacturer's instructions. cDNA was subsequently generated from $1 \mu \mathrm{g}$ of RNA using the Enhanced Avian Reverse Transcriptase kit (Abgene, UK). Levels of podoplanin and CD31 transcripts were estimated using the Ampliflour Uni Primer detection kit (Intergen, UK) and $2 \times$ Q-PCR master mix according to manufacturers instructions. Quantification was carried out using the $\mathrm{iCycler} \mathrm{iQ}^{\mathrm{TM}}$ (Bio-Rad, Hemel Hempstead, UK). Detection of the fluorescence was carried out during the annealing step. Copy numbers for podoplanin and CD31 expression in the samples were determined from in-house standard curves.

\section{Statistical analysis}

Statistical analysis was performed using the Stats Direct statistical software package and the Mann-Whitney $U$ test for continuous variables. All tests were two-tailed with a 5\% significance level used throughout.

\section{RESULTS}

\section{Tumour growth}

There was decreased tumour growth following Celecoxib treatment in both the MCF7/HER2-18 $(P=0.029)$ and MDAMB231 breast cancer cell lines $(P=0.0003)$ (Table 1$)$. These results represent the median values from a total of 43 controls and 41 treated tumours in the MCF7/HER2-18 cell line and 19 controls and 20 treated tumours in the MDAMB231 cell line. The median tumour growth inhibition (TGI) was calculated by comparing the median control growth to the individual treated tumour growth as shown by the equation:

$$
\begin{aligned}
\operatorname{TGI}(\%)= & (1-(\text { individual treated growth } / \\
& \text { median control group growth })) \times 100
\end{aligned}
$$

The median TGI was $58.7 \%$ in the MCF7/HER2-18 tumours and $46.3 \%$ in the MDAMB231 tumours (Table 1). No differences in weight between control and treated mice were seen.

\section{Celecoxib does not perturb cell proliferation}

The differences in tumour growth were not a product of alterations in cell proliferation as there was no change in the median number of proliferating cells in the cell lines between the control and the treated samples as assessed by Ki67 (Table 1). Also, the p21 $1^{\text {cip1 }}$ pathway was not perturbed by the administration of Celecoxib, as there were no significant changes in the median number of cells in each cell line expressing p $21^{\text {cip } 1}$ (Table 1).

\begin{tabular}{|c|c|c|c|c|c|c|}
\hline & \multicolumn{6}{|c|}{ Cell line } \\
\hline & \multicolumn{3}{|c|}{ MCF7 HER2/I 8} & \multicolumn{3}{|c|}{ MDAMB23 I } \\
\hline & Control & Treated & $P$-value & Control & Treated & $P$-value \\
\hline Median growth $\left(\mathrm{mm}^{3}\right)$ & $100.8(38.4-183.5)$ & $39.3(4.0-156.1)$ & 0.029 & $96.4(51.3-284.4)$ & $29.7(0.0-53.3)$ & 0.0003 \\
\hline Median tumour growth inhibition (\%) & \multicolumn{2}{|c|}{$58.7(-7.3-98.2)$} & \multicolumn{4}{|c|}{$46.3(3.7-97.4)$} \\
\hline Mouse weight gain $(g)$ & $0.1(0.7-1.2)$ & $0.8(0.1-1.6)$ & 0.08 & $1.9(1.7-2.0)$ & $2.2(1.7-2.6)$ & 0.22 \\
\hline Median Ki67 (\%) & $55.0(50.9-68.9)$ & $55.5(47.8-63.0)$ & 0.65 & $55.1(47.2-71.6)$ & $57.9(47.7-60.0)$ & 0.91 \\
\hline Median p2 cipl $(\%)$ & $14.5(12.1-17.0)$ & | $6.8(13.1-20.9)$ & 0.07 & $0.6(0.2-2.9)$ & $0.3(0.1-0.3)$ & 0.88 \\
\hline Median TUNEL (\%) & $0.52(0.40-0.63)$ & $0.73(0.58-0.85)$ & 0.0004 & $0.51(0.47-0.82)$ & $0.56(0.343-0.98)$ & 0.96 \\
\hline Median percentage COX-2 positive cells & $65.3(60.3-77.4)$ & $22.5(1.1-60.3)$ & 0.0001 & $26.6(12.0-32.4)$ & $9.4(3.9-18.1)$ & 0.0001 \\
\hline Median COX-2 staining intensity ${ }^{a}$ & $3+$ & $1+$ & 0.046 & - & - & - \\
\hline
\end{tabular}

Table I Tumour growth, proliferation and apoptosis in the cell lines studied

${ }^{a}$ No staining intensity values given for the MDAMB23I cell line as the COX-2 protein was confined to vesicles which could not be quantified by intensity. 


\section{Apoptosis significantly increased following Celecoxib treatment}

There was a significant increase in cell apoptosis in the ER-positive MCF7/HER2-18 cell line following treatment with Celecoxib (median control TUNEL staining $0.52 \%$ (IQR $0.46-0.68$ ), median treated TUNEL staining 0.77 (IQR $0.46-0.68$ ) (Table 1)). There was also an increase in apoptosis in the MDAMB231 cell line that did not reach statistical significance.

\section{Celecoxib inactivates Akt}

Akt (PKB) is a member of the serine/threonine kinases, which in its activated state, is antiapoptotic. Phosphorylation of Akt at the ser473 site was significantly decreased in the MCF7/HER2-18 cell line following treatment with Celecoxib, this was shown at both Western blotting (Figure 1A) and IHC (Figures 2A and B). In the control samples, pAkt ${ }^{\text {ser473 }}$ was found to be in both the cytoplasmic and nuclear areas of the cell, and was distributed throughout the specimen. Celecoxib decreased both the nuclear and cytoplasmic components of $\mathrm{pAkt}^{\text {ser473 }}$ (Figures $2 \mathrm{~A}$ and $\mathrm{B}$ ). The median number of nuclei that stained positive for $\mathrm{pAKT}^{\mathrm{ser} 473}$ dropped from 57.3\% (IQR 54.1-61.1) in the MCF7/HER2-18 controls to $35.7 \%$ (IQR 24.4-47.6\%); $P=0.0001$. In the ERnegative MDAMB-231 cell line, no changes in pAkt ${ }^{\text {ser473 }}$ were seen at either Western blotting (Figure 1B) or IHC (Figure 2C), but as can be seen from the IHC sections, the distribution of $\mathrm{pAkt}^{\mathrm{ser} 473}$ in the MDAMB231 cell line differed from the MCF7/HER2-18 cell line in that there was very low levels of nuclear pAkt ${ }^{\text {ser473 }}$ (Figure 2C). It therefore appears that the subcellular location of $\mathrm{pAkt}^{\mathrm{ser} 473}$ is an important determinant of Celecoxib signalling effect.

\section{COX-2 protein expression}

The pattern of COX-2 expression varied between the cell lines. The MCF7/HER2-18 cell line showed homogeneous cytoplasmic staining across the majority of cells. Celecoxib treatment decreased both the median number of positive cells (median control 65.3\% (IQR 60.3-77.4), median treated 22.5 (IQR 1.1-60.3)) and median staining intensity (Table 1), reflecting a significantly reduced

A

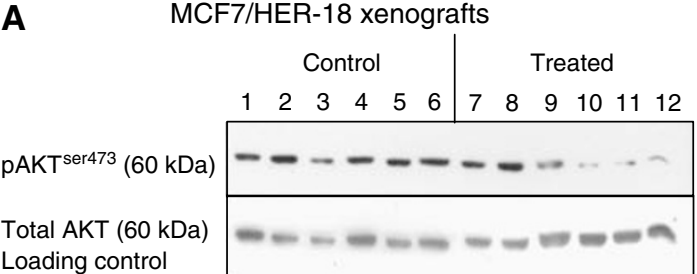

B

MDAMB231 xenografts

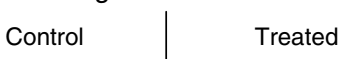

\begin{tabular}{lllllll|lllllll}
1 & 2 & 3 & 4 & 5 & 6 & 7 & 8 & 9 & 10 & 11 & 12 & 13 & 14
\end{tabular}

pAKT $^{\text {ser473 }}(60 \mathrm{kDa})$

Total AKT (60 kDa)

Loading control

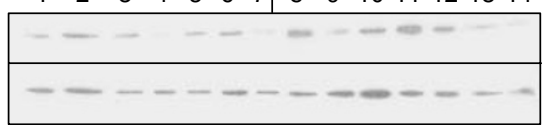

Figure I Western blot MCF7/HER2-18 pAkt ${ }^{\mathrm{ser} 473}$, total Akt. (A) Representative Western blots from MCF7/MCF7/HER2-18 control and Celecoxib-treated tumours. The top blot shows a decrease in PAKT ${ }^{\text {ser473 }}$ in the treated samples $(60 \mathrm{kDa})$. At the bottom, no change was seen in total AKT $(60 \mathrm{kDa})$ as a loading control. The median arbitrary quantity from the control to treated tumours showed a decrease of $28.2 \%$ (IQR $-1.5-$ $89.1 \%$ ) at densitometry over all of the tumours studied. (B) A representative Western blot using lysates from the MDAMB23 I cell line. There was no overall pattern seen. Total AKT was used as a loading control (60 kDa), bottom blot. median immunoscore (Figure 3) of 3 in the treated group, compared to 6 in the control group $(P=0.0001)$. However, the MDAMB231 cell line showed staining that was confined to cytoplasmic vesicles, and therefore staining intensity could not be determined and the scoring was based solely on the number of cells that contained the vesicles. Tumours that had been exposed to Celecoxib again showed a significantly decreased staining for COX-2 protein expression (median control 26.6\% (IQR 12.0-32.4), treated 9.4\% (IQR 3.9-18.1) (Table 1)). In the ER-positive MCF7/ HER2-18 tumours, the percentage of cells that expressed COX-2 in the cytoplasm was significantly correlated to growth; correlation co-efficient $0.4(P=0.02)$.

\section{Lymphangiogenesis}

Q-PCR showed a significant decrease in median podoplanin RNA from the control to the treated tumours. This was seen in both the MCF7/HER2-18 and the MDAMB231 cell lines. The median number of RNA copies per $\mu$ l was 66.61 (IQR 27.87-330.50) in the MCF7/HER2-18 control and fell to 2.94 copies (IQR $0.27-$ 18.50) in the treated tumours $(P=0.05)$ (Table 2). In the MDAMB231 tumours the median number of RNA copies per $\mu \mathrm{l}$ was 160.65 in the control tumours $(2.14-174.75)$ and 0.05 (IQR $0.00-2.99)$ in the treated group $(P=0.015)$. CD31, a marker of angiogenesis also showed reduced numbers of RNA following Celecoxib treatment (Table 2). Our findings were confirmed at the protein level following immunohistochemical staining for podoplanin (Figures 2D-G). At IHC the median number of Chalkley counts in the MCF7/HER2-18 cell line fell from 12 (IQR 9-14) in the control tumours to 9 (IQR 6-10) in the treated samples $(P=0.0001)$, and from 7 (IQR 5-19) in the MDAMB231 tumours, to 4 (IQR 3-6) in the treated samples $(P=0.0001)$ (Table 2$)$. At IHC it could clearly be seen that the majority of lymphatics were in clusters around the periphery of the tissue; therefore, Western blots for podoplanin were not performed.

\section{DISCUSSION}

\section{COX-2 inhibition decreases tumour growth}

High rates of tumour growth and metastasis lead to death from breast cancer. In the nude mice, median tumour growth was inhibited by $58.7 \%$ in the ER-positive MCF7/HER2-18 tumours and by $46.3 \%$ in the ER-negative MDAMB231 tumours, following treatment with Celecoxib. Oestrogen receptor-negative tumours are unresponsive to hormonal manipulation, by way of aromatase inhibitors and tamoxifen, and conventional treatment options are therefore limited. Perturbing the COX-2 signalling pathway may prove to be an important novel therapeutic strategy in both ER-positive and ER-negative tumours reducing the rate of tumour growth.

\section{Celecoxib increases apoptosis via inactivation of Akt}

The mechanism of action of Celecoxib in inhibiting tumour growth was by increasing apoptosis. In the MCF7/HER2-18 cell line, the median number of cells showing apoptosis increased by $40 \%$ from the control to the treated tumours. The fine balance between apoptosis and proliferation is crucial for regulating growth; therefore, even the small numerical changes in apoptosis translate to a large percentage difference, and a significant effect on growth. There were no discernable changes in cell proliferation. This has important implications for current and future trials utilising Celecoxib in breast cancer, as apoptosis, not proliferation should be used as the primary endpoint. Mechanistically, the increase in apoptosis was mediated by inactivation of Akt in the MCF7/HER218 cell line. The decrease in $\mathrm{pAkt}^{\mathrm{ser} 473}$ that we demonstrated was predominantly nuclear with a median decease of nuclear 

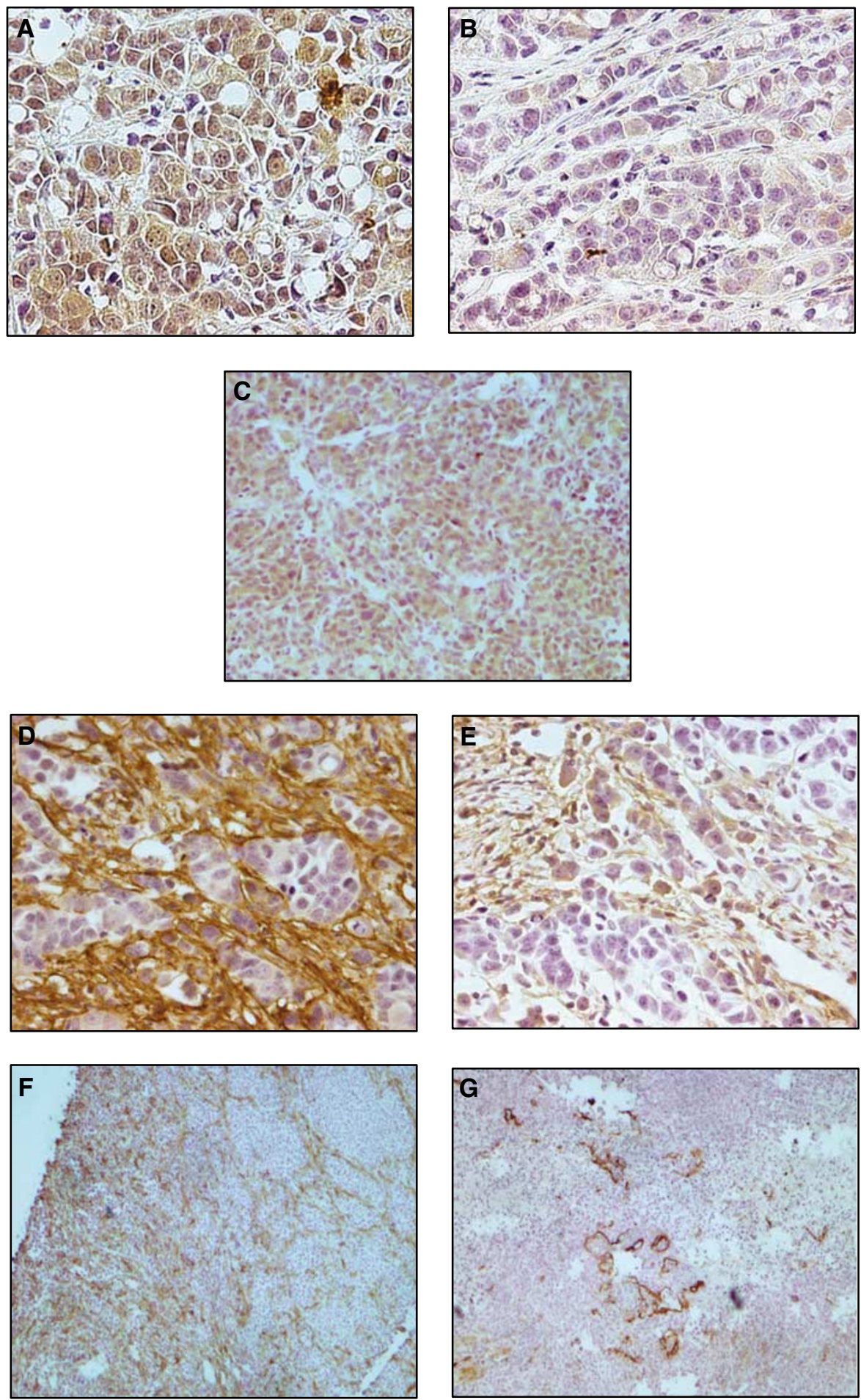

Figure 2 The MCF7/HER2-I 8 control tumours showed expression of pAKT ${ }^{\text {ser } 473}$ in both the cell nucleus and the cell cytoplasm (A). Following Celecoxib treatment both the nuclear and cytoplasmic components of pAKT $T^{\text {ser } 473}$ decreased, this was predominantly seen as a decrease in nuclear pPAKT ${ }^{\text {ser4 } 43}$ (B), which decreased from a median of 57.3\% (IOR 54.I-6I.6) in the control tumours to $35.7 \%$ (IOR 24.4-47.6) in the treated tumours, $P=0.000$ I. The MDAMB23 I cell line expressed predominantly cytoplasmic pAKT $T^{\text {ser473 }}(\mathbf{C})$, which did not significantly decrease following Celecoxib treatment. Decreases in lymphatic vessel density were seen in the MCF7/HER2- I 8 xenografts ( $\mathbf{D}$ and $\mathbf{E})$ from a median of I 2 Chalkley counts to 9 Chalkley counts $(P=0.000$ I). The MDAMB23 I xenografts $(\mathbf{F}$ and $\mathbf{G}$ ) also showed a decrease in podoplanin expression from a median of 7 Chalkley counts to 4 Chalkley counts, $P=0.000 \mathrm{I}$ ) following COX-2 inhibition. Representative IHC staining for pAKT ${ }^{\text {ser } 473}$ and podoplanin in the harvested xenografts: (A) pAKT ${ }^{\text {ser } 473}$ staining in MCF7/HER218 control xenografts $(\times 400) ;(\mathbf{B})$ pAKT ${ }^{\text {ser473 }}$ staining in MCF7/HER2- 8 treated xenografts $(\times 400) ;(\mathbf{C})$ pAKT ${ }^{\text {ser473 }}$ staining in MDAMB23| control xenografts ( $\times$ 400); (D) Podoplanin staining in MCF7/HER2- 8 control xenografts $(\times 400)$; (E) Podoplanin staining in MCF7/HER2- I8 treated xenografts $(\times 400)$; (F) Podoplanin staining in MDAMB23। control xenografts $(\times 100)$; $(\mathbf{G})$ Podoplanin staining in MDAMB23। treated xenografts $(\times 100)$. 
expression from 57 to $36 \%$. Akt (also known as PKB) is a serine threonine kinase that acts downstream of PI3 kinase in the prosurvival pathway. It is thought that activation follows recruitment to the plasma membrane and phosphorylation of both its serine and threonine residues. It has been demonstrated that within $30 \mathrm{~min}$ of activation, Akt detaches from the cytoplasm and relocates to the nucleus. In the nucleus there is modulation of the phosphorylation of a variety of transcription factors (Andjelkovic et al, 1997), including phosphorylation of FKHR1 (Rena et al, 1999) and the forkhead transcription factor AFX (Kops et al, 1999) promoting their cytoplasmic retention - distancing them from their nuclear targets. It has previously been shown that the growth factors PDGF and IGF-1 both cause an increase in the amounts of intra nuclear Akt (Borgatti et al, 2000). There is still, however, some debate as to whether activation occurs solely in the cytoplasm with subsequent translocation to the nucleus, or whether there is a nuclear component to the event itself (Borgatti et al, 2000). The subcellular localisation of pAkt is therefore important for its signalling effect which in the MCF7/HER2-18 cell line was both cytoplamic and nuclear, but predominantly cytoplasmic in the MDAMB231's. This lack of nuclear activated Akt could explain why there was no decrease in phosphorylation seen in the MDAMB231 tumours (and a less substantial increase in apoptosis). Reduced Akt activation following Celecoxib treatment has also been seen in prostate (Hsu et al, 2000) and hepatocellular (Leng et al, 2003) cancer cells. The importance of Akt inactivation in increasing apoptosis was confirmed in PC-3 prostate cancer cells that were engineered to constitutively overexpress Akt which showed reduced apoptosis following Celecoxib treatment compared to cells without constitutively active Akt (Hsu et al, 2000).

\section{Celecoxib reduces lymphatic vessel formation}

Celecoxib reduced levels of the lymphatic endothelial cell marker, podoplanin RNA by $87 \%$ in the MCF7/HER2-18 and $99.9 \%$ in the MDAMB231 tumours. The control samples showed

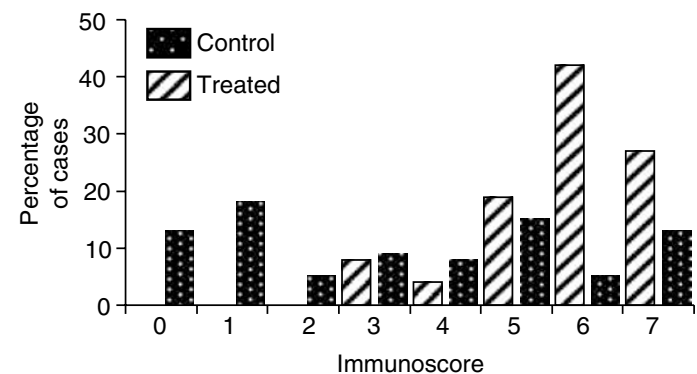

Figure 3 Cyclooxygenase-2 immunoscore in control and Celecoxibtreated MCF7/HER2-I 8 tumours. Cyclooxygenase-2 immunoscore was calculated as the sum of the intensity score $0-4(0=$ none, $\quad I=$ mild, $2=$ moderate, $3=$ strong, $4=$ very strong) and the percent positivity score $(0=$ nil, $1 \leqslant 10 \%, 2=10-50 \%, 3 \geqslant 50 \%)$. No intensity score and therefore immunoscore could be calculated for the MDAMB23I cell line as the staining was confined in cytoplasmic vesicles. variable levels of podoplanin RNA (as seen by the interquartile range values), showing that lymphatic response is specific to the individual tumour/host. Following Celecoxib treatment this range was much less variable, with in some cases a complete lack of expression of podoplanin RNA. The decrease in expression was also seen at a protein level following Chalkley vessel counts; where the lymphatic vessels were predominantly clustered around the tumour periphery. In addition, there was a reduction in the median number of RNA copies of CD31 (an angiogenic marker) by $84 \%$ in the MCF7/HER2-18 and $91 \%$ in the MDAMB231 tumours. The antiangiogenic properties of the COX-2 inhibitors have already been demonstrated (Masferrer et al, 2000). As CD31 and podoplanin RNA were both greatly reduced in the MDAMB231 tumours, this may account for the fact that there was decrease in growth of the MDAMB231 tumours following Celecoxib, even though the increase in apoptosis failed to reach statistical significance. Review of the IHC sample sections showed large areas of central necrosis in the MDAMB231-treated tumours, which again would be consistent with an insufficient blood supply in these Celecoxib-treated tumours.

In breast cancer the primary mode of metastasis is via the lymphatics and COX-2 expression correlates with lymph node metastasis in breast cancer (Ranger et al, 2004). Taken altogether the cumulating data regarding COX-2 inhibition and lymphangiogenesis indicate that there is interesting potential of COX-2 inhibitors being used as antimetastatic agents.

\section{Celecoxib decreases COX-2 protein expression}

Celecoxib treatment decreased the levels of COX-2 protein expression in both cell lines. Therefore, Celecoxib is not simply blocking the effect of COX-2, but is also perturbing COX-2 protein production; a finding noted in previous studies (Cheng et al, 2002; Agarwal et al, 2003). In COX-2 expressing HT29 colon cancer cell lines, COX-2 inhibition with another selective COX-2 inhibitor SC236 (a structural analogue of Celecoxib) showed that at high levels of inhibitor (concentrations greater than $75 \mu \mathrm{m}$ ), COX-2 enzyme activity was completely inactivated. Decreased levels of COX-2 protein expression and increases in apoptosis were seen. Whereas Sulindac sulphide (an inhibitor of COX-1 and COX-2) did not promote any changes in apoptosis and also showed no decrease in COX-2 protein expression) (Agarwal et al, 2003). The antiapoptotic effects of COX-2 were better correlated to decreases in COX-2 protein expression than inhibition of enzymatic activity (Agarwal et al, 2003). It has been suggested that the prostaglandin products of COX-2, mainly PgE2, positively feed back to produce enhanced COX-2 protein expression (Faour et al, 2001). Therefore blocking COX-2, and hence prostaglandin production, would decrease this positive feedback loop. NF $\kappa \mathrm{B}$ also regulates the COX2 gene. Celecoxib abrogates TNF-induced activation of $\mathrm{NF} \kappa \mathrm{B}$ with inhibition of Akt (Shishodia et al, 2004), and therefore another possible explanation for the decrease in COX-2 protein levels seen could be that there is inhibition of $\mathrm{NF} \kappa \mathrm{B}$-induced promoter activity of the COX-2 gene, with subsequent decreases in COX-2 protein expression.

Table 2 Q-PCR for lymphangiogenic (podoplanin) and angiogenic (CD3I) markers and Podoplanin immunohistochemistry (IHC) Chalkley vessel counts

\begin{tabular}{|c|c|c|c|c|c|c|}
\hline Median RNA copy per $\mu$ I (IQR) & \multicolumn{6}{|c|}{ Cell line } \\
\hline 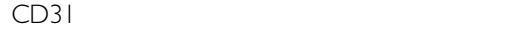 & $47.48(24.34-56.08)$ & $7.70(4.50-21.18)$ & 0.08 & I $40.30(33.84-581.71]$ & $12.87(3.57-59.69)$ & 0.09 \\
\hline Median Podoplanin IHC Chalkley vessel counts & $12(9-14)$ & $9(6-10)$ & 0.0001 & $7(5-19)$ & $4(3-6)$ & 0.0001 \\
\hline
\end{tabular}




\section{COX-2 independent mechanisms of Celecoxib action}

In the ER-positive MCF7/HER2-18 treated tumours, the percentage of residual COX-2 expression significantly correlated with tumour growth, but in the ER-negative MDAMB231-treated tumours, there was no correlation between residual COX-2 expression and growth. This raises the possibility of COX-2 independent effects of Celecoxib in these ER-negative tumours. Further work needs to be carried out to explore the possible COX-2 independent effects of these inhibitors and possible different modes of action in ERpositive $v s$ ER-negative cases. New generation products, which are structurally similar to the COX-2 inhibitors, but lack COX-2 inhibiting effects, are currently under development.

\section{Future direction}

We have shown that Celecoxib significantly decreases breast tumour growth in this nude mouse model and clinical studies are underway to investigate the effect in patient populations. Cyclooxygenase-2 inhibitors are showing great promise for their potential therapeutic benefit in a range of cancers; however there has been recent concern over potential cardiotoxicity. This was due to an increased risk of cardiovascular events following treatment with another COX-2 inhibitor Rofecoxib (Vioxx ${ }^{\mathrm{B}}$ now withdrawn) that was highlighted following the publication of the APPROVe trial (Bombardier et al, 2000; Bresalier et al, 2005). In 2005 the New England Journal of Medicine published a review of Celecoxib cardiac safety which raised concerns over prolonged high-dose COX-2 inhibition (Solomon et al, 2005). A review of the

\section{REFERENCES}

Agarwal B, Swaroop P, Protiva P, Raj SV, Shirin H, Holt PR (2003) Cox-2 is needed but not sufficient for apoptosis induced by Cox-2 selective inhibitors in colon cancer cells. Apoptosis 8: 649-654

Andjelkovic M, Alessi DR, Meier R, Fernandez A, Lamb NJ, Frech M, Cron P, Cohen P, Lucocq JM, Hemmings BA (1997) Role of translocation in the activation and function of protein kinase B. J Biol Chem 272: $31515-31524$

Boland GP, Butt IS, Prasad R, Knox WF, Bundred NJ (2004) COX-2 expression is associated with an aggressive phenotype in ductal carcinoma in situ. Br J Cancer 90: 423-429

Borgatti P, Martelli AM, Bellacosa A, Casto R, Massari L, Capitani S, Neri LM (2000) Translocation of Akt/PKB to the nucleus of osteoblast-like MC3T3-E1 cells exposed to proliferative growth factors. FEBS Lett 477: $27-32$

Bombardier C, Laine L, Reicin A, Shapiro D, Burgos-Vargas R, Davis B, Day R, Ferraz MB, Hawkey CJ, Hochberg HC, Kvien TK, Schnitzer TJ, VIGOR Study Group (2000) Comparison of upper Gastrointestinal toxicity of Rofecoxib and Naproxen in patients with rheumatoid arthritis. $N$ Eng $J$ Med 343: $1520-1528$

Bresalier RS, Sandler RS, Quan H, Bolognese JA, Oxenius B, Horgan K, Lines C, Riddell R, Morton D, Lanas A, Konstam MA, Baron JA (2005) Adenomatous Polyp Prevention on Vioxx (APPROVe) Trial Investigators. N Engl J Med 352: $1092-1107$

Chan KC, Knox WF, Gee JM, Morris J, Nicholson RI, Potten CS, Bundred NJ (2002) Effect of epidermal growth factor receptor tyrosine kinase inhibition on epithelial proliferation in normal and premalignant breast. Cancer Res 62: $122-128$

Cheng HF, Wang CJ, Moeckel GW, Zhang MZ, McKanna JA, Harris RC (2002) Cyclooxygenase-2 inhibitor blocks expression of mediators of renal injury in a model of diabetes and hypertension. Kidney Int 62: 929-939

Connolly EM, Harmey JH, O’Grady T, Foley D, Roche-Nagle G, Kay E, Bouchier-Hayes DJ (2002) Cyclo-oxygenase inhibition reduces tumour growth and metastasis in an orthotopic model of breast cancer. $\mathrm{Br} J$ Cancer 87: $231-237$

Crawford YG, Gauthier ML, Joubel A, Mantei K, Kozakiewicz K, Afshari CA, Tlsty TD (2004) Histologically normal human mammary epithelia with silenced p16(INK4a) overexpress COX-2, promoting a premalignant program. Cancer Cell 5: 263-273 data by Kimmel et al (2005) stated that patients who had been taking Rofecoxib had a three times greater risk of developing a myocardial infarction than patients taking Celecoxib, but the overall safety of Celecoxib is still under debate. As Celecoxib has now been shown to significantly decrease tumour growth, there is an urgency to develop new inhibitors that do not have the potential cardiotoxicity. We must maintain the momentum for further investigation of this class of drug, and its descendents, in relationship to their chemotherapeutic potential and mechanisms of action.

In summary, Celecoxib significantly decreased ER-positive and ER-negative breast tumour growth in our nude mouse xenograft model. The mechanism of action was via inactivation of AKT permitting increased cell death in the ER-positive tumours. The primary mode of breast tumour spread is via the lymphatics. Increasing evidence suggests that COX- 2 has an important role in lymphangiogenesis and we have shown that COX-2 inhibition decreases new lymphatic vessel formation with the potential decrease in metastatic spread of both ER-positive and ER-negative breast tumours.

\section{ACKNOWLEDGEMENTS}

We would like to thank Professor CC Benz for his kind gift of the MCF7/HER2-18 cell line. We would like to thank Pfizer for the donation of the Celecoxib. NLP Barnes was assisted by a Royal College of Surgeons of England Research Grant. This study was funded by an educational grant from Pfizer/Pharmacia.

Davies G, Cunnick GH, Mansel RE, Mason MD, Jiang WG (2004) Levels of expression of endothelial markers specific to tumour-associated endothelial cells and their correlation with prognosis in patients with breast cancer. Clin Exp Metastasis 21: $31-37$

Faour WH, He Y, He QW, de Ladurantaye M, Quintero M, Mancini A, Di Battista JA (2001) Prostaglandin E(2) regulates the level and stability of cyclooxygenase- 2 mRNA through activation of p38 mitogen-activated protein kinase in interleukin-1 beta-treated human synovial fibroblasts. J Biol Chem 276: $31720-31731$

Fu JY, Masferrer JL, Seibert K, Raz A, Needleman P (1990) The induction and suppression of prostaglandin $\mathrm{H} 2$ synthase (cyclooxygenase) in human monocytes. J Biol Chem 265: 16737-16740

Gandhi A, Holland PA, Knox WF, Potten CS, Bundred NJ (2000) Effects of a pure antiestrogen on apoptosis and proliferation within human breast ductal carcinoma in situ. Cancer Res 60: 4284-4288

Gasparini G, Longo R, Sarmiento R, Morabito A (2003) Inhibitors of cyclooxygenase 2: a new class of anticancer agents? Lancet Oncol 4: 605-615

Harris RE, Chlebowski RT, Jackson RD, Frid DJ, Ascenseo JL, Anderson G, Loar A, Rodabough RJ, White E, McTiernan A (2003) Breast cancer and nonsteroidal anti-inflammatory drugs: prospective results from the Women's Health Initiative. Cancer Res 63: 6096-6101

Howe LR, Subbaramaiah K, Patel J, Masferrer JL, Deora A, Hudis C, Thaler HT, Muller WJ, Du B, Brown AM, Dannenberg AJ (2002) Celecoxib, a selective cyclooxygenase 2 inhibitor, protects against human epidermal growth factor receptor 2 (HER-2)/neu-induced breast cancer. Cancer Res 62: $5405-5407$

Hsu AL, Ching TT, Wang DS, Song X, Rangnekar VM, Chen CS (2000) The cyclooxygenase-2 inhibitor celecoxib induces apoptosis by blocking Akt activation in human prostate cancer cells independently of Bcl-2. J Biol Chem 275: $11397-11403$

Kimmel SE, Berlin JA, Reilly M, Jaskowiak J, Kishel L, Chittams J, Strom BL (2005) Patients exposed to Rofecoxib and Celecoxib have different odds of non fatal myocardial infarction. Ann Intern Med 142: $157-164$

Kops GJ, de Ruiter ND, De Vries-Smits AM, Powell DR, Bos JL, Burgering BM (1999) Direct control of the Forkhead transcription factor AFX by protein kinase B. Nature 398: $630-634$

Leng J, Han C, Demetris AJ, Michalopoulos GK, Wu T (2003) Cyclooxygenase-2 promotes hepatocellular carcinoma cell growth 
through Akt activation: evidence for Akt inhibition in celecoxib-induced apoptosis. Hepatology 38: $756-768$

Liu CH, Chang SH, Narko K, Trifan OC, Wu MT, Smith E, Haudenschild C, Lane TF, Hla T (2001) Overexpression of cyclooxygenase-2 is sufficient to induce tumorigenesis in transgenic mice. J Biol Chem 276: $18563-18569$

Masferrer JL, Leahy KM, Koki AT, Zweifel BS, Settle SL, Woerner BM, Edwards DA, Flickinger AG, Moore RJ, Seibert K (2000) Antiangiogenic and antitumor activities of cyclooxygenase-2 inhibitors. Cancer Res 60: $1306-1311$

Ranger GS, Thomas V, Jewell A, Mokbel K (2004) Elevated cyclooxygenase2 expression correlates with distant metastases in breast cancer. Anticancer Res 24: 2349-2351

Rena G, Guo S, Cichy SC, Unterman TG, Cohen P (1999) Phosphorylation of the transcription factor forkhead family member FKHR by protein kinase B. J Biol Chem 274: 17179-17183

Roche-Nagle G, Connolly EM, Eng M, Bouchier-Hayes DJ, Harmey JH (2004) Antimetastatic activity of a cyclooxygenase-2 inhibitor. $\mathrm{Br} J$ Cancer 91: 359-365

Shishodia S, Koul D, Aggarwal BB (2004) Cyclooxygenase (COX)-2 inhibitor celecoxib abrogates TNF-induced NF-kappa B activation through inhibition of activation of I kappa B alpha kinase and Akt in human non-small cell lung carcinoma: correlation with suppression of COX-2 synthesis. J Immunol 173: 2011-2022
Slamon DJ, Clark GM, Wong SG, Levin WJ, Ullrich A, McGuire WL (1987) Human breast cancer: correlation of relapse and survival with amplification of the HER-2/neu oncogene. Science 235: 177-182

Smith WL, DeWitt DL, Garavito RM (2000) Cyclooxygenases: structural, cellular, and molecular biology. Annu Rev Biochem 69: 145-182

Solomon SD, McMurray JJ, Pfeffer MA, Wittes J, Fowler R, Finn P, Anderson WF, Zauber A, Hawk E, Bertagonolli M, Adenoma prevention with Celecoxib (APC) study investigators (2005) Cardiovascular risk associated with Celecoxib in a clinical trial for Colorectal Adenoma Prevention. $N$ Eng J Med 352: $1071-1080$

Sposto R, Silverstein MJ (2002) Predicting local recurrence in pateints with ductal carcinoma in situ. In Ductal Carcinoma In Situ of the Breast, M] Silverstein (ed) Chapter 21, pp. 255-263. Lippncott, Williams and Wilkins: Philadelphia, USA

Su JL, Shih JY, Yen ML, Jeng YM, Chang CC, Hsieh CY, Wei LH, Yang PC, Kuo ML (2004) Cyclooxygenase-2 induces EP1- and HER-2/Neu-dependent vascular endothelial growth factor-C up-regulation: a novel mechanism of lymphangiogenesis in lung adenocarcinoma. Cancer Res 64: 554-564

Subbaramaiah K, Telang N, Ramonetti JT, Araki R, DeVito B, Weksler BB, Dannenberg AJ (1996) Transcription of cyclooxygenase-2 is enhanced in transformed mammary epithelial cells. Cancer Res 56: 4424-4429

Xie WL, Chipman JG, Robertson DL, Erikson RL, Simmons DL (1991) Expression of a mitogen-responsive gene encoding prostaglandin synthase is regulated by mRNA splicing. Proc Natl Acad Sci USA 88: 2692-2696 